\title{
Effusion under the microscope
}

\author{
S.G. Chong1, Z. Chauhan², E. Di Nino2, A.O. Brien1, B.P. Casserly1
}

ABSTRACT: Effusion under the microscope. S.G. Chong, Z. Chauhan, E. Di Nino, A.O. Brien, B.P. Casserly.

Pseudochylothorax is a very rare form of pleural effusion. It is also called chyliform or cholesterol pleural effusion. It is usually a unilateral process and approximately one-third of patients are asymptomatic at presentation. We report a case of a 60 year old man with a background of rheumatoid arthritis who presented with progressive dyspnea. Chest X-ray revealed a new left pleural effusion and a small persistent right pleural effusion. He presented 5 years prior due to recurrent pleural effusion and no diagnosis was made. Repeat thoracentesis yielded 350 milliliters of thick, milky, tan-colored fluid.

Monaldi Arch Chest Dis 2012; 77: 1, 32-34.

Keywords: Pleural effusion, Pseudochylothorax, Chylothorax, Rheumatoid arthritis.

1 Mid-Western Regional Hospital, Dooradoyle, Limerick, Ireland,

2 Memorial Hospital Of Rhode Island, Brown University, Providence, Rhode Island, USA.

Correspondence: Sy Giin Chong, Respiratory Department, Mid-Western Regional Hospital, Dooradoyle, Limerick, Ireland; e-mail address: sygiin@hotmail.com

\section{Case Report}

A 60 year old gentleman presented with progressively worsening dyspnoea of 8 days in duration. This was associated with a non-productive cough. There was no associated symptoms. There was no history of recent travel. His past medical history was significant for a 60-pack-year smoking history, rheumatoid arthritis diagnosed 6 years prior, chronic leg edema and lower extremity ulcers secondary to chronic venous changes. He was treated previously for his rheumatoid arthritis but the treatment was stopped due to poor compliance.

Five years prior, the patient underwent thoracentesis for a recurrent right pleural effusion which revealed milky pleural fluid and a diffusely nodular and thickened pleura. Giant multinucleated histiocytes were noted in the pleural fluid at that time. A year later, he underwent a left anterior thoracotomy with pericardiectomy for a large pericardial effusion. No diagnosis were made at both occasions.

On admission, he was apyrexial and his vitals were stable. He appeared in mild respiratory distress. There was no jugular venous distention and no stridor. Rheumatoid nodules was noted on both of his elbows and there was evidence of clinical synovitis on both of his hands. His chest examination revealed a left thoracotomy scar, diminished lung sounds and dullness to percussion at both bases. Cardiac examination was normal. In the lower extremities there was bilateral pitting edema, skin thickening, and venous stasis.

Bloods on admission showed a WBC (white blood cell) count of $12.4 \times 10^{3} / \mathrm{uL}$ with $81 \%$ neutrophils, a Hgb (haemoglobin) of $12.7 \mathrm{~g} / \mathrm{dL}$, and a platelet count of $327 \times 103 / \mu \mathrm{L}$. Chemistry values were normal. B-type natriuretic peptide and tro- ponin concentration were normal. Rheumatoid factor was 1:640 and anti-citrullinated peptide antibodies was positive. A chest X-Ray showed a new left pleural effusion and an unchanged small right pleural effusion compared to a previous chest X-Ray done 6 months earlier. A CT (computed tomography) scan revealed bilateral nodular pleural thickening in addition to the pleural effusions (figure 1). A thoracocentesis was performed which yielded 350 milliliters of thick, milky, tan-colored fluid (figure 2). Initial gram stain demonstrated many polymorphonuclear leukocytes, with moderate intra- and extra-cellular gram positive cocci in pairs and chains. Analysis of the pleural fluid revealed a WBC $68,000 \times 10^{-3} / \mu \mathrm{L}, \mathrm{RBC} 49,000 \times 10^{-3} / \mu \mathrm{L}$, total pro-

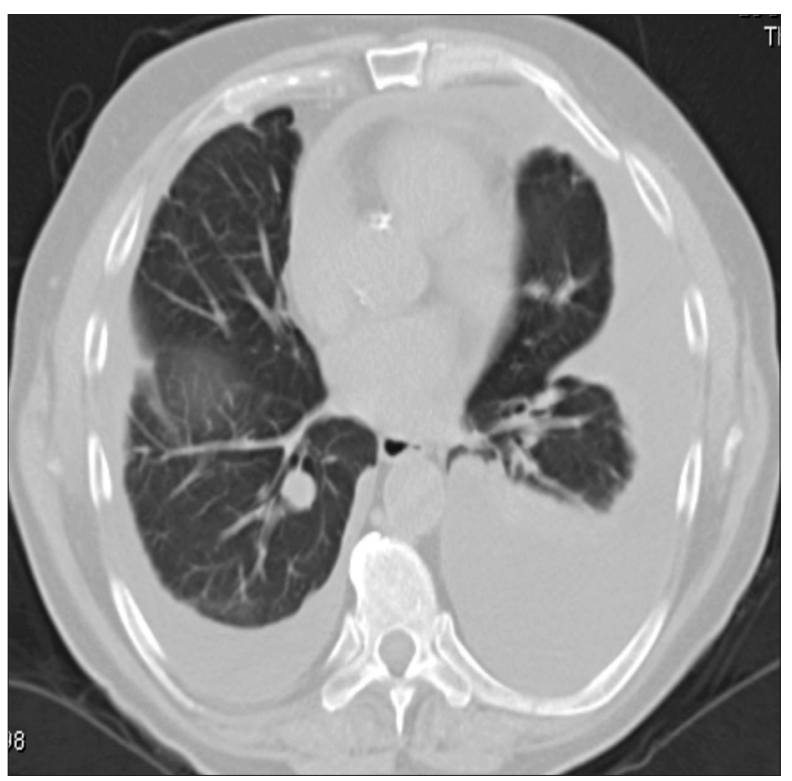

Fig. 1. - Bilateral nodular pleural thickening with pleural effusion seen at CT. 


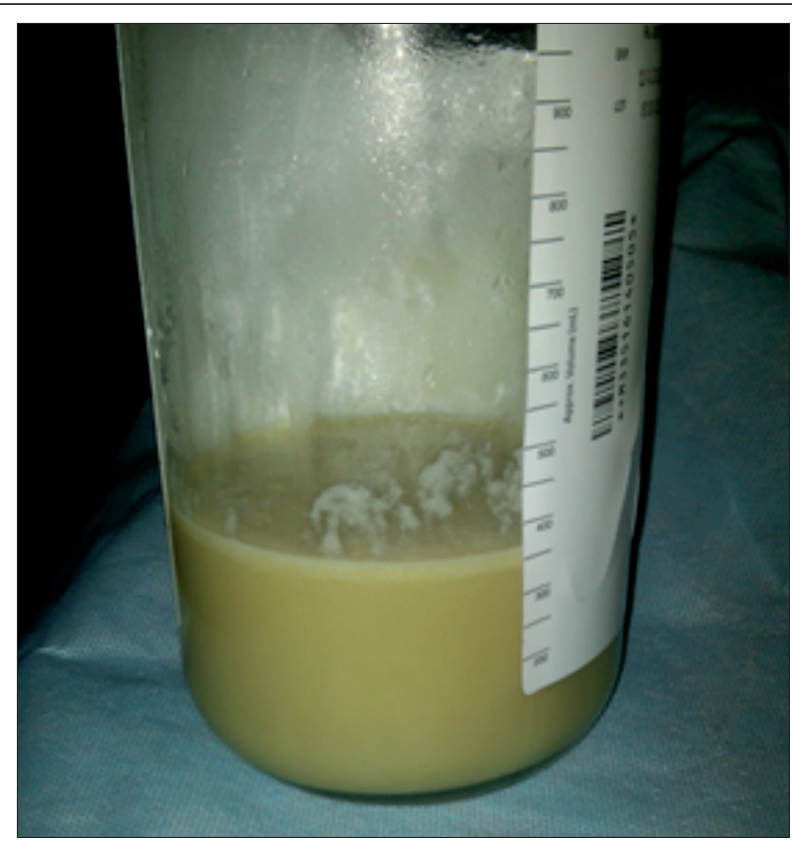

Fig. 2. - Thick milky, tan coloured pleural effusion.

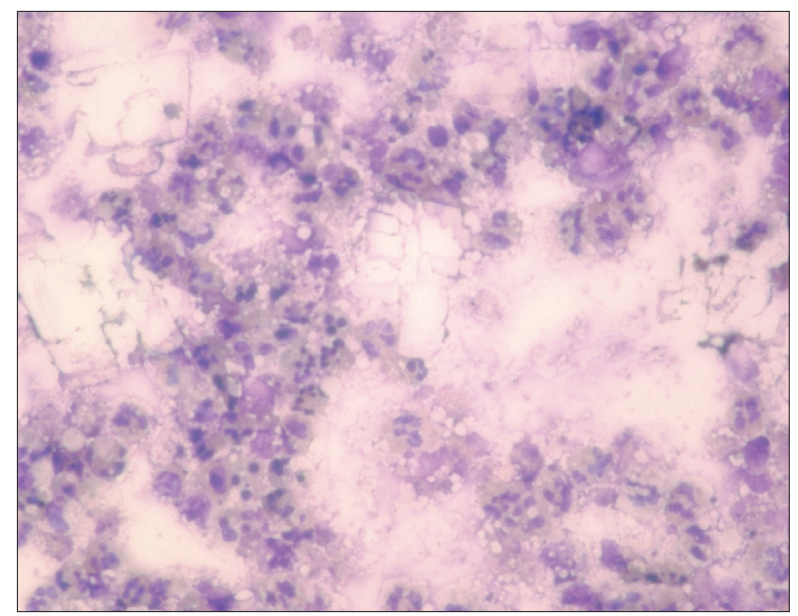

Fig. 3. - Pleural effusion under microscope showing numerous cholesterol crystals.

tein $4.6 \mathrm{~g} / \mathrm{dL}$, glucose $0.17 \mathrm{mmol} / \mathrm{L}$, LDH (lactate dehydrogenase) 18,954 IU/L, pH 7.043, cholesterol $153 \mathrm{mg} / \mathrm{dL}$, triglycerides $248 \mathrm{mg} / \mathrm{dL}$, cholesterol to triglyceride ratio 0.62, AFB (acid-fast bacilli) smear was negative and negative pleural fluid chylomicrons. Serum LDH measured 117 IU/L, serum total protein $7.1 \mathrm{~g} / \mathrm{dL}$, and a glucose of $7.6 \mathrm{mmol} / \mathrm{L}$. Microscopic examination of the pleural fluid is shown below and revealed the numerous cholesterol crystals (figure 3).

He was diagnosed with pseudochylothorax effusion secondary to poorly controlled rheumatoid arthritis, now complicated by infection. Despite the cholesterol to triglyceride ratio of less than one, a chylous effusion was excluded by lipoprotein electrophoresis showing an absence of chylomicrons.

\section{Discussion}

Pseudochylothorax is a pleural effusion that has a high concentration of cholesterol and is com- monly associated with long standing inflammatory disorders such as tuberculosis or rheumatoid arthritis [1]. Pseudochylothorax effusions may develop after an exudative effusion remains in the chest chronically, leading to pleural thickening and fibrosis [2]. Patients with pseudochylothorax typically have radiographic evidence of thickened or calcified pleural surfaces, as in this case. However, this traditional view has been recently challenged by the finding of pseudochylothorax effusions in patients without thickened pleura, and without long standing effusions [3].

Microscopic and chemical evaluation of the pleural fluid establishes the diagnosis of pseudochylothorax. Microscopic examination may reveal cholesterol crystals in pseudochylothorax, but the absence of this finding does not exclude the diagnosis [4]. Chemical analysis of pseudochylothorax often demonstrates elevated cholesterol concentrations greater than $250 \mathrm{mg} / \mathrm{dL}$ and a triglyceride concentration below $110 \mathrm{mg} / \mathrm{dL}$ [5]. This is as opposed to a chylothorax, where the triglyceride concentration is above this level [1]. Chylomicrons are not present in a pseudochylothorax.

Previously published literature also states that pseudochylothorax effusions can be diagnosed using a cholesterol to triglyceride ratio of greater than one and that this value is generally felt to be inviolable $[6,7]$. However, our patient's ratio was less than one. Cholesterol crystals, which likely result from supersaturation, can occur in effusions that at the time of diagnosis do not have high cholesterol levels. Cholesterol crystals may be present with pleural cholesterol levels as low as 150 $\mathrm{mg} / \mathrm{dL}$ and not be visualized with pleural cholesterol levels as high as $800 \mathrm{mg} / \mathrm{dL}$ [6]. This suggests that the cholesterol levels which initially may be elevated can subsequently vary with disease evolution.

The putative mechanism by which cholesterol accumulates in the pleural space involves the lysis of blood cells, which release free cholesterol from their cell membrane [8]. Pleural inflammation and fibrosis impair lymphatic drainage causing this cholesterol and other molecules to collect within the pleural space. Occasionally the cholesterol concentration is high enough for crystals to precipitate out of the effusion, as evidenced in this patient. In the setting of active pleural space inflammation, pseudochylothorax can lead to visceral pleural restriction.

However, the report of 6 patients with pseudochylothorax but without pleural thickening casts doubt on the aforementioned mechanism of cholesterol accumulation. One possible explanation is put forth in a study which shows that lowdensity lipoprotein-bound cholesterol accumulates in the pleural space in acute inflammatory effusions, whereas high-density lipoproteins are mostly found in chronic inflammatory effusions [9]. This suggests that local metabolic processes cause in part the elevated cholesterol concentration, not the degradation of erythrocytes and white blood cells. 


\section{References}

1. Staats BA, Ellefson RD, Budahn LL, Dines DE, Prakash UB, Offord K, The lipoprotein profile of chylous and nonchylous pleural effusions. Mayo Clin Proc 1980; 55: 700-4.

2. Garcia-Zamalloa A, Ruiz-Irastorza G, Aguayo FJ, et al. Pseudochylothorax: report of 2 cases and review of the literature. Medicine (Baltimore) 1999; 78: 200207.

3. Wrightson JM, Stanton AE, Maskell NA, Davies RJ, Lee YC, Pseudochylothorax without pleural thickening: time to reconsider pathogenesis? Chest 2009; 136: $1144-7$.
4. Hillerdal G. Chyliform (cholesterol) pleural effusion. Chest 1985; 88: 426-8.

5. Coe JE, Aikawa JK, Cholesterol pleural effusion. Arch Intern Med 1961; 108: 163-174.

6. Agrawal V, Sahn SA. Lipid Pleural Effusions. Am J Med Sci 2008; 335: 16-20.

7. Coe JE, Aikawa JK. Cholesterol pleural effusion. Report of 2 cases studied with isotopic techniques and review of the world literature. Arch Intern Med 1961; 108: 163-174.

8. Ferguson GC. Cholesterol pleural effusion in rheumatoid lung disease. Thorax 1966; 21: 577-582.

9. Hamm H, Pfalzer B, Fabel H. Lipoprotein analysis in a chyliform pleural effusion: implications for pathogenesis and diagnosis. Respiration 1991; 58: 294-300.

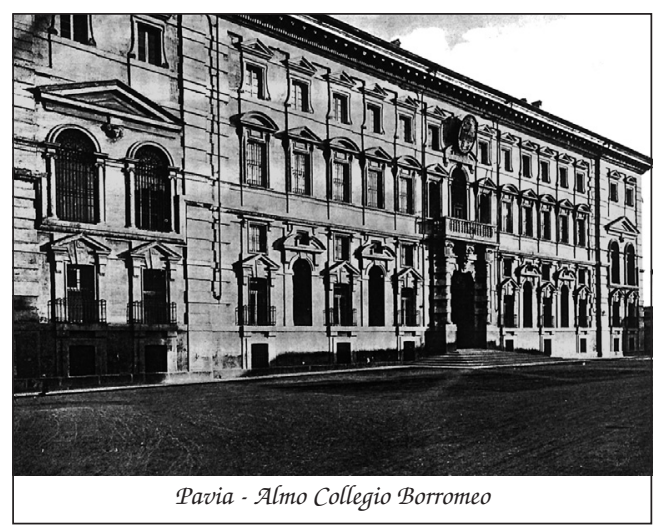

